\title{
Molière Re-Envisioned. Twenty-First Century Retakes./ Renouveau et renouvellement moliéresques. Reprises contemporaines, dir. M.J. MURATORE
}

\section{Laura Rescia}

\section{(2) OpenEdition}

\section{Edizione digitale}

URL: https://journals.openedition.org/studifrancesi/31668

DOI: $10.4000 /$ studifrancesi.31668

ISSN: 2421-5856

\section{Editore}

Rosenberg \& Sellier

\section{Edizione cartacea}

Data di pubblicazione: 1 août 2020

Paginazione: 400-401

ISSN: 0039-2944

\section{Notizia bibliografica digitale}

Laura Rescia, «Molière Re-Envisioned. Twenty-First Century Retakes./ Renouveau et renouvellement moliéresques. Reprises contemporaines, dir. M.J. MURATORE», Studi Francesi [Online], 191 (LXIV | II) | 2020, online dal 01 septembre 2020, consultato il 16 septembre 2021. URL: http://journals.openedition.org/ studifrancesi/31668; DOI: https://doi.org/10.4000/studifrancesi.31668

Questo documento è stato generato automaticamente il 16 septembre 2021.

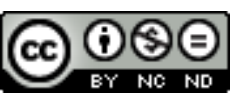

Studi Francesi è distribuita con Licenza Creative Commons Attribuzione - Non commerciale - Non opere derivate 4.0 Internazionale. 


\title{
Molière Re-Envisioned. Twenty-First Century Retakes./ Renouveau et renouvellement moliéresques. Reprises contemporaines, dir. M.J. MURATORE
}

\author{
Laura Rescia
}

\section{NOTIZIA}

Molière Re-Envisioned. Twenty-First Century Retakes./ Renouveau et renouvellement moliéresques. Reprises contemporaines, dir. M.J. MURATORE, Paris, Hermann, 2018, 633 pp.

1 L'ambizioso obiettivo di questo volume bilingue, come dichiarato dalla curatrice nella prefazione, è raccogliere nuove prospettive critiche sull'opera molieresca, rivisitando le influenze socio-politiche che ne hanno determinato gli sviluppi, e offrendo nuove analisi testuali e linguistiche, che si avvalgano dell'ottica contemporanea. Gli studiosi che hanno collaborato al volume, una trentina, provengono da tradizioni critiche differenti (francese, inglese, statunitense e italiana), e presentano un largo spettro di approcci diversi e complementari. Per brevità, non riportiamo il dettaglio del lungo indice, limitandoci a indicare qualche elemento che caratterizza ogni sezione del libro. Sono quattro gli assi privilegiati, che organizzano il raggruppamento degli articoli. Dopo un saggio introduttivo di ALAIN VIALA, Molière homme de spectacle, si apre la prima parte, dedicata a saggi di carattere storico, filologico e linguistico: JAN CLARKE esamina le conseguenze economiche dei viaggi a Corte di Molière, con uno studio quantitativo basato sui registri di La Grange; LAURA NAUDEIX propone il concetto di "poétique de la présence" per designare sia la corporeità del palcoscenico molieresco, sia la capacità di creare un rapporto con il pubblico, grazie alla possibilità di una lettura "a chiave" dei personaggi e delle situazioni sceniche; questo stesso approccio viene problematizzato da LAURENT DUBREUIL in relazione al rapporto tra Les précieuses ridicules e il contesto 
storico-sociale a cui allude; ALAIN REY dimostra come l'uso delle strategie linguistiche adottate dal drammaturgo gli abbia permesso di diventare un classico; anche CLAUDINE NÉDÉLEC e CÉLINE PARINGAUX adottano un'ottica linguistica per affrontare in particolare il linguaggio amoroso e la capacità del drammaturgo di destreggiarsi tra registri linguistici e linguaggi di specialità; CONSTANTIN FROSIN formula delle proposte sull'origine dei nomi Tartuffe, su cui si focalizza anche il successivo contributo di PHILIPPE LE PAPE, e di Molière.

2 La seconda parte è dedicata agli studi teorici e relativi ai generi letterari: MITCHELL GREENBERG affronta la questione della comicità nel Tartuffe e nell'Avare, per esplorarne l'ambigua funzione, fra trasgressione e conservatorismo; LOUIS UCCIANI problematizza la questione del contributo di Molière allo sviluppo della commedia moderna; RENÉ CORONA esplora la scrittura di Molière interrogando i concetti di comicità, barocco e modernità; MARIE-CLAUDE CANOVA-GREEN analizza le caratteristiche de Les Fâcheux e di altre comédieballets, che valuta come genere ibrido, ma pur sempre con la supremazia del testo sulla musica, ancora concepita come semplice ornamento; GIOVANNI DOTOLI commenta Dom Juan utilizzando la categoria del burlesco.

3 Una terza parte del volume racchiude gli articoli dedicati alle analisi testuali: CHARLES MAZOUER affronta la questione della religione dei personaggi di Molière; NICHOLAS HAMMOND analizza la funzione delle canzoni nelle sue commedie; RALPH ALBANESE rintraccia i segni della scarsità alimentare della Francia secentesca nel Dom Juan e nell'Avare; MARY-JO MURATORE rileva a partire dal Dom Juan una progressiva inflessione della ragione, che verrebbe incrinata da una vena di follia, così come la morale da un irrompere del piacere; PIERRE BRUNEL offre una lettura della scena del Povero nel Dom Juan; ed è ancora questa commedia al centro del contributo di MARCELLA LEOPIZZI, che analizza i personaggi femminili, tra debolezze e forza di carattere; THERESA VARNEY KENNEDY allarga il punto di vista sulla "questione femminile" nell'intera opera del drammaturgo; PERRY GETHNER analizza come i salons vengano descritti nelle commedie di Molière e in quelle delle scrittrici coeve, trovando punti di contatto, come la critica della pedanteria, ma altresì di divergenza: Molière sembra adottare un punto di vista più prossimo alla realtà dei salotti, mentre le autrici descrivono un mondo in cui le donne si muovono fuori dalla logica patriarcale; ILDA THOMAS adotta l'ottica comparata, analizzando il trattamento riservato ai personaggi maschili ne L'École des Femmes e nei Rusteghi di Goldoni.

L'ultima parte è dedicata all'influenza sui secoli successivi e alle riletture contemporanee: JEFF PERSEL analizza il testo Molière 2.0, un adattamento realizzato negli Stati Uniti a scopo divulgativo, che condensa venti commedie in una sola pièce in due atti, messa in scena nel 2015 e disponibile in Rete; ENCARNATIÒN MEDINA ARJONA propone una panoramica su personaggi, tipi e comicità nella poetica del drammaturgo; GIOVANNA DEVINCENZO esamina alcune riprese delle Précieuses ridicules, nelle messe in scena francesi dal 2007 al 2010; JOSEPH HARRIS si concentra sulla ricezione del Misanthrope nella Lettre à d'Alembert di Rousseau, e più in generale sul tema della misantropia nell'opera rousseauiana; MICHAEL S. KOPPISCH si focalizza sulle presenze dell'opera molieriana negli scritti di Charlotte Delbo; CONCETTA CAVALLIN esamina la riscrittura del mito di Anfitrione in Molière e Giraudoux; SOPHIE BASTIEN esamina Le Malade imaginaire, suggerendo che almeno cinque aspetti del trattamento del tema della medicina, appaiono estremamente vicini alla contemporaneità (abuso di farmaci, logica del 
profitto nei trattamenti medici e farmacologici, effetti secondari della farmacopea, presunzione del sapere medico, terapie alternative), oltre a tre tematiche psicologiche (l'ipocondria, opportunismo dei parenti del malato, narcisismo dei medici). 